\title{
Evaluation of peripheral fundus autofluorescence in eyes with wet age-related macular degeneration
}

This article was published in the following Dove Press journal:

Clinical Ophthalmology

14 December 2016

Number of times this article has been viewed

\section{Tetsuyuki Suetsugu ${ }^{1,2}$ \\ Aki Kato' \\ Munenori Yoshida' \\ Tsutomu Yasukawa' \\ Akiko Nishiwaki ${ }^{1,3}$ \\ Norio Hasegawa' \\ Hideaki Usui ${ }^{1}$ \\ Yuichiro Ogura'}

'Department of Ophthalmology and Visual Science, Nagoya City University Graduate School of Medical Sciences,

${ }^{2}$ Department of Ophthalmology, General Kamiiida Daiichi Hospital,

${ }^{3}$ Nishiwaki Eye Clinic, Nagoya,

Aichi, Japan
Correspondence: Aki Kato

Department of Ophthalmology and Visual Science, Nagoya City University Graduate School of Medical Sciences, I Kawasumi, Mizuho-cho, Mizuho-ku, Nagoya,

Aichi 467-860 I, Japan

Tel +81528538251

Fax +8I 52 84I 9490

Email akikato@med.nagoya-cu.ac.jp
Purpose: We aimed to evaluate the prevalence of abnormal peripheral fundus autofluorescence (FAF) in wet age-related macular degeneration (AMD) using wide-field imaging instrument.

Patients and methods: A retrospective, case-controlled study involving 66 eyes of 46 Japanese wet AMD patients and 32 eyes of 20 control patients was performed. Wide-field FAF images were obtained for typical AMD (37 eyes/28 patients), polypoidal choroidal vasculopathy (PCV) (22 eyes/20 patients), and retinal angiomatous proliferation (RAP) (seven eyes/four patients). Two masked ophthalmologists independently graded the images for mottled, granular, and nummular patterns. Main outcome measures were abnormal peripheral FAF frequencies and relative risks by disease subgroups and treatments.

Results: Abnormal peripheral FAF patterns were found in 51.5\% of wet AMD eyes compared with $18.8 \%$ of control eyes $(P<0.001)$. Mottled, granular, and nummular patterns were found in $45.5 \%, 31.8 \%$, and $16.7 \%$, respectively, of wet AMD eyes. Each disease subgroup (typical AMD, $54.1 \%$; PCV, 36.4\%; and RAP, $85.7 \%$ ) showed significantly higher frequencies of peripheral FAF $(P<0.001, P=0.03$, and $P<0.001$, respectively) than control eyes $(18.8 \%)$. There were no significant differences $(P=0.76)$ between the frequencies in untreated and treated eyes.

Conclusion: Eyes of Japanese wet AMD patients had a higher abnormal FAF prevalence compared with control eyes. Among the three disease subtypes, abnormal patterns were least prevalent in PCV eyes.

Keywords: age-related macular degeneration, fundus autofluorescence, polypoidal choroidal vasculopathy, retinal angiomatous proliferation

\section{Introduction}

Age-related macular degeneration (AMD) is currently the leading cause of blindness in the industrialized world. ${ }^{1-5}$ Choroidal neovascularization often causes severe and rapid visual loss in the wet or neovascular form of AMD, while geographic atrophy gradually impairs the central vision in the dry or atrophic type of AMD. It is important to understand age-related changes in retinal pigment epithelial (RPE) cells under the sensory retina, because the changes predispose eyes to development of AMD.

Fundus autofluorescence (FAF) imaging is a noninvasive technique used to assess RPE abnormalities, which are observed often in aging eyes and eyes with AMD, retinitis pigmentosa, and other chorioretinal diseases. FAF signals increase with lipofuscin accumulation in RPE cells and decrease with RPE atrophy. Analysis of FAF is an effective method to observe the functions of the RPE cells, which are related closely to the pathogenesis of wet AMD. ${ }^{6-11}$

Previous research into AMD has focused primarily on the posterior pole (central $30^{\circ}-50^{\circ}$ around the macula). Currently, a wide-field imaging instrument, the Optos ${ }^{\circledR} 200$ Tx (Optos plc, Dunfermline, Scotland, UK), allows noninvasive, 
nonmydriatic scanning of the fundus, including the periphery. ${ }^{12-17}$ Some studies have reported abnormal peripheral FAF in patients with AMD examined using the wide-field retinal imaging technique. ${ }^{18-21}$

The purpose of the current study was to characterize the frequencies of abnormal peripheral FAF patterns in the eyes of Japanese patients with AMD with respect to AMD disease types, treatments, and locations.

\section{Patients and methods Study model}

This was a retrospective, case-control study conducted in Nagoya City University Hospital. The institutional review board of Nagoya City University Graduate School of Medical Sciences approved the retrospective data collection and analysis (University hospital Medical Information Network [UMIN] identification number: UMIN000018746).

\section{Ethical approval}

All procedures performed in studies involving human participants were in accordance with the ethical standards of the institutional and/or national research committee and with the 1964 Declaration of Helsinki and its later amendments or comparable ethical standards.

\section{Informed consent}

All patients provided written informed consent for use of their medical data in research.

\section{Inclusion and exclusion criteria}

Patients with wet AMD included in the study had been diagnosed with typical AMD, polypoidal choroidal vasculopathy (PCV), and retinal angiomatous proliferation (RAP). Control eyes included in the study were mainly normal eyes or eyes with mild cataract without other diagnosed abnormalities. Normal fellow eyes of patients with wet or dry AMD, eyes with central serous chorioretinopathy, and eyes with three or more obscure peripheral FAF quadrants were excluded from the study.

\section{Patient characteristics}

The mean patient ages of the three wet AMD disease subgroups, namely, typical AMD, PCV, and RAP, are shown in Table 1 . There were no significant $(P=0.07)$ differences between the mean ages of the patients with wet AMD (76 \pm 6.1 years) and those with the control eyes ( $72 \pm 7.6$ years). There also were no significant $(P=0.09, P=0.24$, and $P=0.19$, respectively) differences among the mean patient ages in
Table I Characteristics of the study group

\begin{tabular}{llll}
\hline Group & Patients & Eyes & Mean age, years \\
\hline Control & 20 & 32 & $72 \pm 7.6$ \\
All wet AMD & 46 & 66 & $76 \pm 6.1$ \\
Typical AMD & 28 & 37 & $76 \pm 5.7$ \\
PCV $^{a}$ & 20 & 22 & $75 \pm 6.5$ \\
RAP $^{a}$ & 4 & 7 & $78 \pm 6.1$ \\
\hline
\end{tabular}

Note: ${ }^{\mathrm{D}}$ Disease subgroups within the wet AMD group.

Abbreviations: AMD, age-related macular degeneration; PCV, polypoidal choroidal vasculopathy; RAP, retinal angiomatous proliferation.

the typical AMD, PCV, and RAP subgroups compared with the ages of patients with control eyes. All patients were Japanese.

\section{Image analysis}

Wide-field FAF images obtained using the Optos 200Tx were collected and analyzed. The central $30^{\circ}$ region of the retina was covered in each image. Each $90^{\circ}$ section of the peripheral images was divided into superior, inferior, temporal, and nasal quadrants (Figure 1). Two masked ophthalmologists (MY, TY) graded the wide-field FAF images from the eyes with wet AMD and the control eyes in random order. The images were graded independently in each quadrant for the presence of three abnormal peripheral FAF patterns, ie, mottled, granular, and nummular (Figures 2-4), according to a previous report. ${ }^{20}$ If the images were obscured, the results were reported as undeterminable. A third masked ophthalmologist grader (AK) resolved any discrepancies; in these cases, the matches between one of the first two graders and the third grader were adopted as the final gradings. If several types of abnormal peripheral FAF patterns were observed in the same quadrant, these were all recorded. If none of the

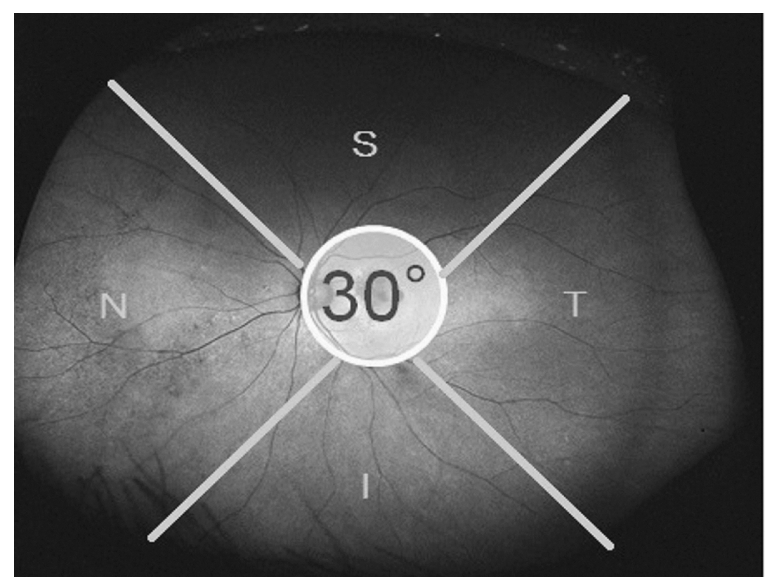

Figure I Optos ${ }^{\circledR}$ image of an eye divided into four quadrants.

Notes: The central $30^{\circ}$ region of the retina was covered, and each $90^{\circ}$ section of the peripheral image was divided into the following four quadrants: S, superior; I, inferior; N, nasal; and T, temporal. 


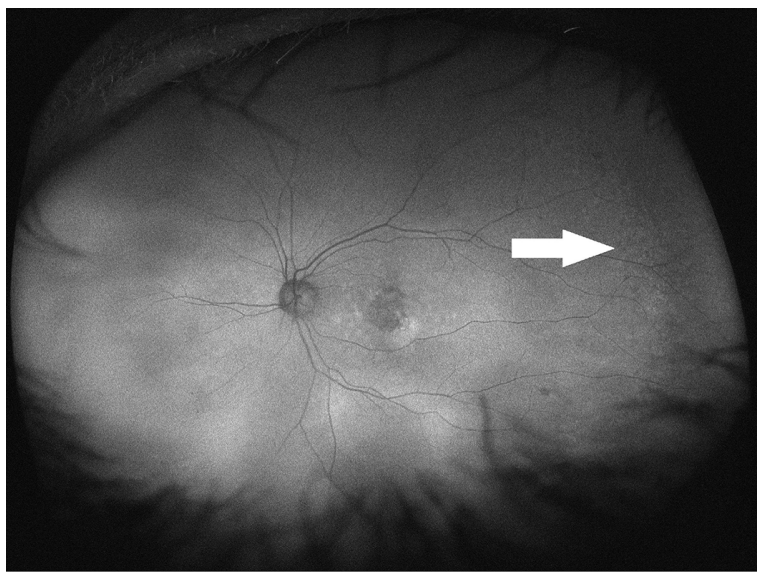

Figure 2 Optos ${ }^{\circledR}$ image of an eye with mottled pattern from a wet age-related macular degeneration patient.

Note: A mottled pattern is indicated by an arrow, characterized by a diffuse area of decreased irregular autofluorescence.

three graders agreed, the image was graded as having no abnormal pattern.

\section{Main outcome measures}

The main outcome measures were abnormal peripheral FAF frequencies and relative risks by disease subgroups and treatments.

\section{Statistical analysis}

Statistical analyses were conducted using Microsoft Excel 2010 (Microsoft Corporation, Redmond, WA, USA). Normal distribution of the mean ages in all groups was confirmed using the Jarque-Bera (JB) test after obtaining skewness and kurtosis values with Excel functions and calculating the JB values using its formula. The calculations were confirmed by

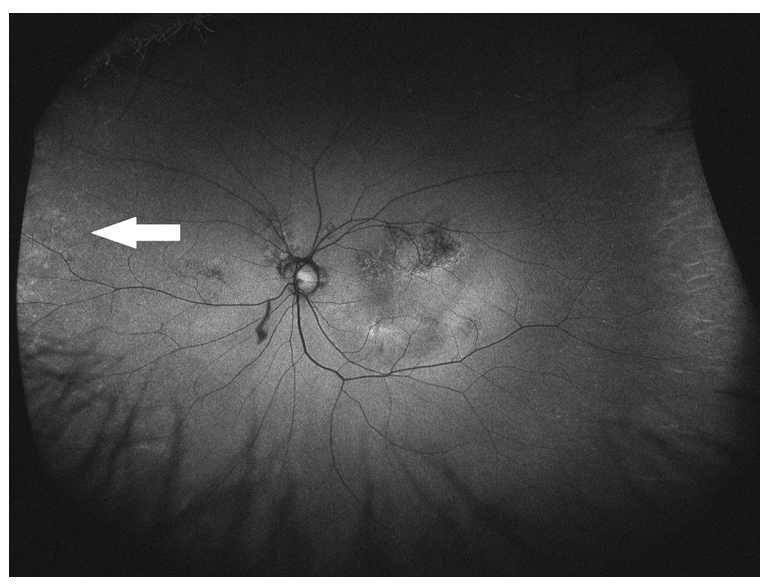

Figure 3 Optos $^{\circledR}$ image of an eye with granular pattern from a wet age-related macular degeneration patient.

Note: A granular pattern is indicated by an arrow, characterized by a small, interspersed area of increased autofluorescence.

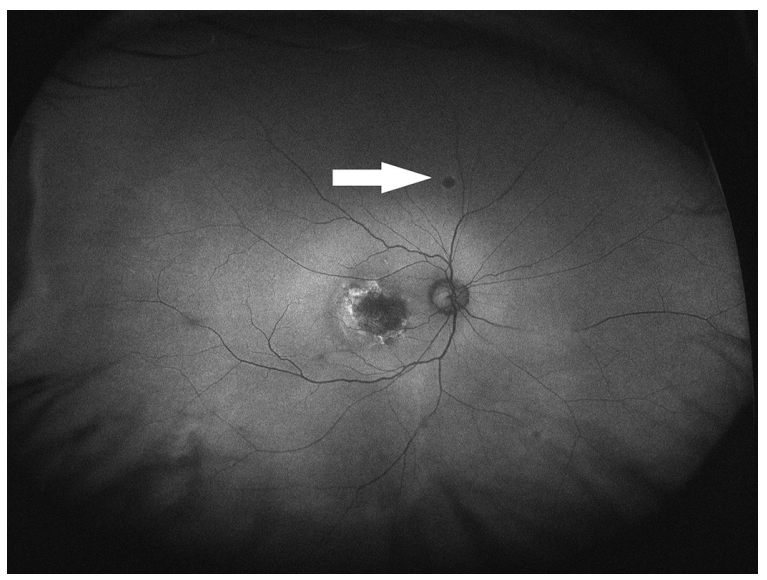

Figure 4 Optos $^{\circledR}$ image of an eye with nummular pattern from a wet age-related macular degeneration patient.

Note: A nummular pattern is indicated by an arrow, characterized by a small, isolated homogeneous area of decreased autofluorescence.

MATLAB $^{\circledR}$ (MathWorks, Natick, MA, USA) software, and the same results were obtained. $F$-tests for age distribution analyses, unpaired $t$-tests for mean age comparisons, and chi-square tests for frequency comparisons were performed. In all analyses, $P<0.05$ was considered significant.

\section{Results}

\section{Prevalence of abnormal peripheral FAF}

Abnormal peripheral FAF was evident in 34 (51.5\%) eyes with wet AMD and six (18.8\%) control eyes, a difference that reached significance $(P<0.001)$. The prevalence rates of abnormal peripheral FAF patterns in all wet AMD and control groups are shown in Table 2. Abnormal patterns observed in descending order were mottled, granular, and nummular. The agreement between two graders was $\kappa=0.17$ for the types of abnormal FAF patterns. Table S1 shows the reasons for grading disagreements between any two ophthalmologists.

\section{Relative risk analyses by wet AMD disease types}

The relative risk analyses of the frequencies of abnormal peripheral FAF patterns by wet AMD disease types are shown

Table 2 Frequencies of abnormal peripheral FAF patterns in wet AMD eyes

\begin{tabular}{llllll}
\hline FAF type & \multicolumn{2}{l}{ Frequencies, \% } & OR & $\mathbf{9 5 \% ~ C l ~}$ & P-value \\
\cline { 2 - 4 } & All wet AMD & Control & & & \\
\hline Mottled & 45.5 & 18.8 & 3.6 & $1.6-7.9$ & $<0.001$ \\
Granular & 31.8 & 6.3 & 7.0 & $2.3-21.5$ & $<0.001$ \\
Nummular & 16.7 & 6.3 & 3.0 & $0.9-9.8$ & $<0.001$ \\
\hline
\end{tabular}

Abbreviations: AMD, age-related macular degeneration; $\mathrm{Cl}$, confidence interval; FAF, fundus autofluorescence; $O R$, odds ratio. 
Table 3 Relative risk analyses of abnormal peripheral FAF by wet AMD disease types

\begin{tabular}{lllll}
\hline Group & $\begin{array}{l}\text { Frequencies of abnormal } \\
\text { peripheral FAF, \% }\end{array}$ & OR & 95\% Cl & P-value \\
\hline Control & 18.8 & 1.0 & - & - \\
All wet AMD & 51.5 & 4.6 & $1.7-12.6$ & $<0.00 \mathrm{I}$ \\
Typical AMD $^{\text {a }}$ & 54.1 & 5.1 & $1.7-15.3$ & $<0.00 \mathrm{I}$ \\
PCV $^{\text {a }}$ & 36.4 & 2.5 & $0.7-8.6$ & 0.03 \\
RAP $^{\mathrm{a}}$ & 85.7 & 26.0 & $2.6-258.2$ & $<0.00 \mathrm{I}$ \\
\hline
\end{tabular}

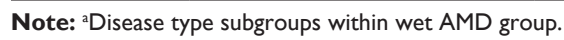

Abbreviations: AMD, age-related macular degeneration; FAF, fundus autofluorescence; OR, odds ratio; $\mathrm{Cl}$, confidence interval; PCV, polypoidal choroidal vasculopathy; RAP, retinal angiomatous proliferation.

in Table 3. Abnormal FAF patterns were most prevalent in eyes with RAP, intermediate in eyes with typical AMD, and least prevalent in eyes with PCV.

\section{Prevalence of abnormal peripheral FAF patterns based on wet AMD disease types}

The prevalence rates of abnormal peripheral FAF patterns in each wet AMD disease type are shown in Table 4. The mottled pattern was seen most often among all disease types. The granular pattern was seen most often in eyes with RAP.

\section{Relative risk analysis of abnormal peripheral FAF by wet AMD treatments}

The relative risk analyses of the frequencies of abnormal peripheral FAF patterns based on the wet AMD treatments are shown in Table 5. Of the 66 eyes with wet AMD studied, $24(36.4 \%)$ eyes were untreated and 42 eyes $(63.6 \%)$ were treated with intravitreal aflibercept injections $\left(\right.$ Eylea $^{\circledR}$; Regeneron Pharmaceuticals Inc, Tarrytown, NY, USA) (22 eyes), intravitreal ranibizumab injections (Lucentis ${ }^{\circledR}$; Genentech, South San Francisco, CA, USA) (29 eyes), and photodynamic therapy (PDT) with verteporfin injection (Visudyne ${ }^{\circledR}$;

Table 4 Frequencies of abnormal peripheral FAF in each wet AMD disease type

\begin{tabular}{llllll}
\hline FAF type & \multicolumn{4}{l}{$\begin{array}{l}\text { Number of eyes with abnormal pattern } \\
\text { (\% of total of number eyes in group) }\end{array}$} \\
\cline { 2 - 6 } & Control & $\begin{array}{l}\text { All wet } \\
\text { AMD }\end{array}$ & $\begin{array}{l}\text { Typical } \\
\text { AMD }^{\mathbf{a}}\end{array}$ & PCV $^{\mathbf{a}}$ & RAP $^{\mathbf{a}}$ \\
\hline Mottled & $6(18.8)$ & $30(45.5)$ & $18(48.6)$ & $7(3 \mathrm{I} .8)$ & $5(71.4)$ \\
Granular & $2(6.3)$ & $21(31.8)$ & $12(32.4)$ & $6(27.3)$ & $3(42.9)$ \\
Nummular & $2(6.3)$ & II (16.7) & $6(16.2)$ & $4(18.2)$ & $1(14.3)$ \\
Total eyes & 32 & 66 & 37 & 22 & 7 \\
\hline
\end{tabular}

Note: aDisease type subgroups within wet AMD group.

Abbreviations: AMD, age-related macular degeneration; FAF, fundus autofluorescence; PCV, polypoidal choroidal vasculopathy; RAP, retinal angiomatous proliferation.
Table 5 Relative risk analyses of abnormal peripheral FAF by wet AMD treatments

\begin{tabular}{lllll}
\hline $\begin{array}{l}\text { Treatment } \\
\text { type }\end{array}$ & $\begin{array}{l}\text { Frequencies of abnormal } \\
\text { peripheral FAF, \% }\end{array}$ & OR & $\mathbf{9 5 \%} \mathbf{C l}$ & $\boldsymbol{P}$-value \\
\hline Untreated & 50.0 & 1.0 & - & - \\
Treated & 52.4 & 1.1 & $0.4-3.0$ & 0.76 \\
IVA $^{a}$ & 40.9 & 0.7 & $0.2-2.2$ & 0.39 \\
IVR $^{a}$ & 58.6 & 1.4 & $0.5-4.2$ & 0.35 \\
PDT $^{a}$ & 58.3 & 1.4 & $0.3-5.7$ & 0.56 \\
\hline
\end{tabular}

Note: ${ }^{\text {TT }}$ reatment subgroups within treated group.

Abbreviations: AMD, age-related macular degeneration; FAF, fundus autofluorescence; $\mathrm{Cl}$, confidence interval; IVA, intravitreal aflibercept injection; IVR, intravitreal ranibizumab injection; OR, odds ratio; PDT, photodynamic therapy.

Novartis International AG, Basel, Switzerland) (12 eyes). There were no significant differences in the frequencies of the abnormal FAF patterns between each treated group and the untreated group, ie, $50.0 \%$ in untreated eyes and 52.4\% in treated eyes.

\section{Prevalence of abnormal peripheral FAF in each image quadrant}

The prevalence rates of abnormal peripheral FAF in each image quadrant are shown in Table 6. Abnormal peripheral FAF was most prevalent in the nasal quadrant and least prevalent in the superior quadrant. Eight (12.1\%) eyes with wet AMD had images that were obscured in the superior quadrant, six (9.1\%) eyes in the inferior quadrant, two (3.0\%) eyes in the temporal quadrant, and one (1.5\%) eye in the nasal quadrant.

\section{Discussion}

In this study, we observed abnormal peripheral FAF in 34 eyes $(51.5 \%)$ in the group of eyes with wet AMD in Japanese patients. Compared with the prevalence (six eyes, $18.8 \%$ ) in the healthy control eye group, the prevalence was significantly higher in eyes with wet AMD. The types of abnormal peripheral FAF observed were mottled (45.5\%), granular (31.8\%), and nummular (16.7\%). The frequencies of abnormal peripheral FAF patterns were highest in eyes with RAP (85.7\%), intermediate in eyes with typical AMD (54.1\%), and lowest in eyes with PCV (36.4\%). The

Table 6 Frequencies of abnormal peripheral FAF patterns in each image quadrant

\begin{tabular}{lllll}
\hline Eye type & \multicolumn{4}{l}{ Frequencies of abnormal peripheral FAF, \% } \\
\cline { 2 - 5 } & Superior & Inferior & Temporal & Nasal \\
\hline All wet AMD & 3.0 & 22.7 & 22.7 & 40.9 \\
Control & 6.3 & 12.5 & 12.5 & 12.5 \\
P-values & 0.20 & 0.01 & 0.02 & $<0.00$ I \\
\hline
\end{tabular}

Abbreviations: AMD, age-related macular degeneration; FAF, fundus autofluorescence. 
treatments did not affect the frequencies of the abnormal peripheral FAF patterns in eyes with wet AMD. To the best of our knowledge, the previous findings regarding abnormal peripheral FAF patterns in patients with wet AMD were reported mainly in Caucasian patients, ${ }^{19-21}$ while only a few findings were described solely in Asian patients. ${ }^{18}$

Tan et $\mathrm{al}^{20}$ and Nomura et $\mathrm{al}^{18}$ previously reported that the frequencies of abnormal peripheral FAF in patients with wet AMD were $86.0 \%$ and $60.4 \%$, respectively. Although dry and wet AMD were not differentiated, Witmer et $\mathrm{al}^{21}$ and Heussen et $\mathrm{al}^{12}$ reported $63.6 \%$ and $73.9 \%$, respectively, as the frequencies of the abnormal peripheral FAF patterns in eyes with AMD. The current report found lower incidence rates compared with these previous reports. However, in the current study, the increase of FAF abnormalities in eyes with wet AMD was significant compared with control eyes. The previous studies did not report differences in the frequencies among different AMD types or the effect of AMD treatments on the frequencies of abnormal FAF patterns.

Japanese populations are characterized by higher rates of PCV and lower rates of RAP compared with Western populations. The prevalence rates of PCV in Japanese populations with AMD range from $23 \%$ to about $55 \%,{ }^{22-25}$ while they range from $9 \%$ to about $17 \%$ in Caucasian populations with AMD. ${ }^{22,26,27}$ In contrast, the prevalence of RAP has been reported as $4.5 \%$ in a Japanese population with AMD,${ }^{25}$ while the rates range from $13.5 \%$ to about $15 \%$ in a Caucasian population with AMD. ${ }^{22,28}$ In the current study, more than a third of the eyes had PCV. Japanese populations have tended to have more eyes with PCV, and the frequency of abnormal peripheral FAF patterns is lower in PCV eyes than in eyes with other disease types. Such ethnic characteristics may have contributed to the lower total number of eyes with abnormal FAF observed in the current study.

Previous studies have reported three major patterns of the abnormal peripheral FAF. ${ }^{18,20}$ First, the mottled pattern of decreased FAF was characterized by diffuse areas of irregular hypoautofluorescence, which corresponded to RPE depigmentation in $76.5 \%$ of eyes with wet AMD, according to Tan et al. ${ }^{20}$ In a study of the fellow eyes of Japanese patients with unilateral wet AMD, Sasaki et $\mathrm{al}^{29}$ reported that retinal pigment abnormalities without drusen were critical in the development of PCV. In the current study, the mottled pattern was found in $31.8 \%$ of PCV eyes; this abnormality may be associated closely with RPE depigmentation.

Second, the granular pattern of increased FAF was characterized by small, interspersed areas of hyperautofluorescence that corresponded to peripheral drusen in $82.7 \%$ of eyes with wet AMD, according to Tan et al. ${ }^{20}$ The drusen in Japanese eyes with wet AMD was reported to be associated more typically with RAP than with PCV, and these were observed in $77 \%$ to $\sim 95 \%$ of eyes with RAP $^{25,30}$ and in $19 \%$ to $~ 24 \%$ of eyes with PCV. ${ }^{25,31}$ The drusens were identified easily as increased autofluorescence in wide-field FAF images. The presence of drusen may have contributed to a higher frequency of abnormal FAF in eyes with RAP. In the current study, the drusen corresponding to the granular FAF pattern was observed in $42.9 \%$ of eyes with RAP.

Finally, the nummular pattern of decreased FAF was characterized by small-to-intermediate areas of homogeneous hypoautofluorescence that corresponded to RPE atrophy with a cobblestone appearance in $69.8 \%$ of eyes with wet AMD, according to Tan et al. ${ }^{20}$

The current study also analyzed the frequency of abnormal peripheral FAF relative to treatments and locations. Intravitreal injections of anti-vascular endothelial growth factor and PDT treatments did not affect the rates of abnormal peripheral FAF patterns, perhaps because of the marginal effect of these therapies on the development of FAF abnormalities. The current study also showed that abnormal FAF developed less often in the superior quadrant, which may be due to disruption by artifacts such as eyelashes and eyelids, which result in more obscured images in the superior quadrant than in other quadrants.

The evaluation of abnormal peripheral FAF in eyes with wet AMD may lead to development of potent treatment strategies based on abnormal FAF patterns. Different types of treatments may be administered to treat mottled, granular, and nummular patterns in subsequent studies. The prognoses based on factors such as visual acuity, number of treatments, and quality of life also should be analyzed. The efficacy of the treatments may be analyzed by wide-field FAF images acquired at different time points. Studies of differential treatment strategies based on the abnormal peripheral FAF patterns may lead to novel treatment approaches for wet AMD.

The current study had some limitations. First, the data were collected retrospectively and did not consider the time period after the onset of wet AMD. The treatment types and periods during which they were administered varied among the patients. Second, we did not perform multivariate analyses of the effects of age, sex, smoking, time length after wet AMD onset, and number of treatments. Multivariate analyses may yield some valuable findings about the prevalence of abnormal peripheral FAF in patients with wet AMD. Third, we did not evaluate the healthy fellow eyes in patients with wet AMD. The fellow eyes of patients with wet AMD may 
have more abnormal peripheral FAF compared with the eyes of healthy control patients because a previous study reported a high concordance rate $(90 \%)$ in both eyes of patients with wet AMD. ${ }^{20}$ Finally, fewer patients were recruited compared with other similar studies. The results may have differed if we had recruited more patients with wet AMD.

\section{Conclusion}

The eyes of Japanese patients with wet AMD had higher prevalence rates of abnormal peripheral FAF in typical AMD, PCV, and RAP subgroups compared with control eyes. Mottled, granular, and nummular patterns were all observed in the eyes with wet AMD, and the mottled pattern was seen most frequently. Associations between abnormal peripheral FAF with the treatments and the prognostic factors should be investigated further in prospective clinical investigations.

\section{Acknowledgment}

No author has received any financial support.

\section{Disclosure}

The authors report no conflicts of interest in this work.

\section{References}

1. Congdon N, O'Colmain B, Klaver CC, et al; Eye Diseases Prevalence Research Group. Causes and prevalence of visual impairment among adults in the United States. Arch Ophthalmol. 2004;122(4):477-485.

2. Maberley DA, Hollands H, Chuo J, et al. The prevalence of low vision and blindness in Canada. Eye (Lond). 2006;20(3):341-346.

3. Wong TY, Loon SC, Saw SM. The epidemiology of age related eye diseases in Asia. Br J Ophthalmol. 2006;90(4):506-511.

4. Xu L, Wang Y, Li Y, et al. Causes of blindness and visual impairment in urban and rural areas in Beijing: the Beijing Eye Study. Ophthalmology. 2006;113(7):1134.e1-1134.e11.

5. Owen CG, Jarrar Z, Wormald R, Cook DG, Fletcher AE, Rudnicka AR. The estimated prevalence and incidence of late stage age related macular degeneration in the UK. Br J Ophthalmol. 2012;96(5):752-756.

6. Bindewald A, Bird AC, Dandekar SS, et al. Classification of fundus autofluorescence patterns in early age-related macular disease. Invest Ophthalmol Vis Sci. 2005;46(9):3309-3314.

7. Dandekar SS, Jenkins SA, Peto T, et al. Autofluorescence imaging of choroidal neovascularization due to age-related macular degeneration. Arch Ophthalmol. 2005;123(11):1507-1513.

8. Delori FC, Fleckner MR, Goger DG, Weiter JJ, Dorey CK. Autofluorescence distribution associated with drusen in age-related macular degeneration. Invest Ophthalmol Vis Sci. 2000;41(2):496-504.

9. Holz FG, Bellman C, Staudt S, Schutt F, Volcker HE. Fundus autofluorescence and development of geographic atrophy in age-related macular degeneration. Invest Ophthalmol Vis Sci. 2001;42(5):1051-1056.

10. Holz FG, Bindewald-Wittich A, Fleckenstein M, Dreyhaupt J, Scholl HP, Schmitz-Valckenberg S; FAM-Study Group. Progression of geographic atrophy and impact of fundus autofluorescence patterns in age-related macular degeneration. Am J Ophthalmol. 2007;143(3):463-472.

11. Schmitz-Valckenberg S, Bindewald-Wittich A, Dolar-Szczasny J, et al; Fundus Autofluorescence in Age-Related Macular Degeneration Study Group. Correlation between the area of increased autofluorescence surrounding geographic atrophy and disease progression in patients with AMD. Invest Ophthalmol Vis Sci. 2006;47(6):2648-2654.
12. Heussen FM, Tan CS, Sadda SR. Prevalence of peripheral abnormalities on ultra-widefield greenlight (532 nm) autofluorescence imaging at a tertiary care center. Invest Ophthalmol Vis Sci. 2012;53(10):6526-6531.

13. Heussen FM, Vasconcelos-Santos DV, Pappuru RR, Walsh AC, Rao NA, Sadda SR. Ultra-wide-field green-light (532-nm) autofluorescence imaging in chronic Vogt-Koyanagi-Harada disease. Ophthalmic Surg Lasers Imaging. 2011;42(4):272-277.

14. Ogura S, Yasukawa T, Kato A, et al. Wide-field fundus autofluorescence imaging to evaluate retinal function in patients with retinitis pigmentosa. Am J Ophthalmol. 2014;158(5):1093-1098.

15. Oishi A, Ogino K, Makiyama Y, Nakagawa S, Kurimoto M, Yoshimura N. Wide-field fundus autofluorescence imaging of retinitis pigmentosa. Ophthalmology. 2013;120(9):1827-1834.

16. Reznicek L, Seidensticker F, Stumpf C, et al. Systematic analysis of wide-field fundus autofluorescence (FAF) imaging in posterior uveitis. Curr Eye Res. 2014;39(2):164-171.

17. Seidensticker F, Neubauer AS, Wasfy T, et al. Wide-field fundus autofluorescence corresponds to visual fields in chorioretinitis patients. Clin Ophthalmol. 2011;5:1667-1671.

18. Nomura Y, Takahashi H, Tan X, Obata R, Yanagi Y. Widespread choroidal thickening and abnormal midperipheral fundus autofluorescence characterize exudative age-related macular degeneration with choroidal vascular hyperpermeability. Clin Ophthalmol. 2015;9:297-304.

19. Reznicek L, Wasfy T, StumpfC, et al. Peripheral fundus autofluorescence is increased in age-related macular degeneration. Invest Ophthalmol Vis Sci. 2012;53(4):2193-2198.

20. Tan CS, Heussen F, Sadda SR. Peripheral autofluorescence and clinical findings in neovascular and non-neovascular age-related macular degeneration. Ophthalmology. 2013;120(6):1271-1277.

21. Witmer MT, Kozbial A, Daniel S, Kiss S. Peripheral autofluorescence findings in age-related macular degeneration. Acta Ophthalmol. 2012; 90(6): $428-\mathrm{e} 433$.

22. Coscas G, Yamashiro K, Coscas F, et al. Comparison of exudative agerelated macular degeneration subtypes in Japanese and French patients: multicenter diagnosis with multimodal imaging. Am J Ophthalmol. 2014;158(2):309.e-318.e.

23. Mori R, Yuzawa M, Akaza E, Haruyama M. Treatment results at 1 year of ranibizumab therapy for polypoidal choroidal vasculopathy in eyes with good visual acuity. Jpn J Ophthalmol. 2013;57(4):365-371.

24. Sho K, Takahashi K, Yamada H, et al. Polypoidal choroidal vasculopathy: incidence, demographic features, and clinical characteristics. Arch Ophthalmol. 2003;121(10):1392-1396.

25. Maruko I, Iida T, Saito M, Nagayama D, Saito K. Clinical characteristics of exudative age-related macular degeneration in Japanese patients. Am J Ophthalmol. 2007;144(1):15-22.

26. Hatz K, Prunte C. Polypoidal choroidal vasculopathy in Caucasian patients with presumed neovascular age-related macular degeneration and poor ranibizumab response. Br J Ophthalmol. 2014;98(2):188-194.

27. Scassellati-Sforzolini B, Mariotti C, Bryan R, Yannuzzi LA, Giuliani M, Giovannini A. Polypoidal choroidal vasculopathy in Italy. Retina. 2001; 21(2):121-125.

28. Donati MC, Carifi G, Virgili G, Menchini U. Retinal angiomatous proliferation: association with clinical and angiographic features. Ophthalmologica. 2006;220(1):31-36.

29. Sasaki M, Kawasaki R, Uchida A, et al. Early signs of exudative agerelated macular degeneration in Asians. Optom Vis Sci. 2014;91(8): 849-853.

30. Sawa M, Ueno C, Gomi F, Nishida K. Incidence and characteristics of neovascularization in fellow eyes of Japanese patients with unilateral retinal angiomatous proliferation. Retina. 2014;34(4):761-767.

31. Mori K, Horie-Inoue K, Gehlbach PL, et al. Phenotype and genotype characteristics of age-related macular degeneration in a Japanese population. Ophthalmology. 2010;117(5):928-938. 


\section{Supplementary material}

Table SI Reasons for grading disagreements between two ophthalmologists

\begin{tabular}{lll}
\hline Categories & Number of disagreements & $\%$ \\
\hline Unclear image & 107 & 49.3 \\
Finding granular pattern & $\mathrm{I}$ & 0.5 \\
Finding nummular pattern & $\mathrm{I}$ & 0.5 \\
Finding mottled pattern & 86 & 39.6 \\
Two patterns in same quadrant & $\mathrm{II}$ & 5.1 \\
Distinguish mottled vs nummular or granular pattern & $\mathrm{II}$ & 5.1 \\
Total & 217 & 100 \\
\hline
\end{tabular}

\section{Publish your work in this journal}

Clinical Ophthalmology is an international, peer-reviewed journal covering all subspecialties within ophthalmology. Key topics include: Optometry; Visual science; Pharmacology and drug therapy in eye diseases; Basic Sciences; Primary and Secondary eye care; Patient Safety and Quality of Care Improvements. This journal is indexed on

Submit your manuscript here: http://www.dovepress.com/clinical-ophthalmology-journal
Dovepress

PubMed Central and CAS, and is the official journal of The Society of Clinical Ophthalmology (SCO). The manuscript management system is completely online and includes a very quick and fair peer-review system, which is all easy to use. Visit http://www.dovepress.com/ testimonials.php to read real quotes from published authors. 